\title{
A Hardware Simulator for the Generation of Ultrasonic Transmission Test Signals
}

\author{
Michael Vogt ${ }^{1}$, Martin Gevers ${ }^{2}$, Rogier Geerders ${ }^{3}$, Jan Förster ${ }^{2}$ \\ ${ }^{1}$ KROHNE Messtechnik GmbH, Ludwig-Krohn-Str. 5, 47058 Duisburg, Germany, \\ ${ }^{2}$ KROHNE Innovation GmbH, Ludwig-Krohn-Str. 5, 47058 Duisburg, Germany, \\ ${ }^{3}$ KROHNE New Technologies BV, Kerkeplaat 12, 3313 LC Dordrecht, The Netherlands \\ m.vogt@krohne.com
}

\begin{abstract}
Summary:
The quality and characteristics of the transmission signals in ultrasonic transit-time flowmeters are important with regard to the accuracy of the time of flight and the flow measurements. In this contribution, a hardware simulator for generating ultrasonic transmission signals is presented, which allows for testing the sensor electronics of the flowmeter at zero flow and under well-defined and highly reproducible conditions. The concept is to replace the liquid by a block of polymethylmethacrylate as ultrasound propagation medium. The resulting effects on the transmission signal are analyzed and discussed.
\end{abstract}

Keywords: Ultrasonic flowmeter, testing, transmission attenuation, time of flight

\section{Introduction}

In ultrasonic transit-time flowmeters, ultrasound waves are propagated downstream and upstream a flowing fluid (liquid or gas). Based on the measured times of flight (TOFs), the volumetric flow rate is calculated. The ultrasound transducers play an important role for the time domain characteristics (amplitude, shape, duration, etc.) of the transmission signals. Because various operating conditions (pressure, temperature, etc.) have to be considered in the transducer design, the signal characteristics is largely transducer-specific. For testing of the sensor electronics, realistic transmission signal measurements are required. The below presented hardware simulator has been designed for performing test measurements at zero flow under well-defined and highly reproducible conditions.

\section{Concept of the Hardware Simulator}

In Fig. 1 a), the transmission path of an ultrasonic transit-time flowmeter containing a liquid is illustrated. Two transducers are positioned opposite to each other with the flowing liquid (SOS: speed of sound $c_{\mathrm{L}}, \mathrm{Al}$ : acoustic impedance $Z_{\mathrm{L}}$, AC: attenuation coefficient $\alpha_{\mathrm{L}}(f)$ ) in-between. The concept of the hardware simulator in Fig. $1 \mathrm{~b}$ ) is to replace the heavy flowmeter and the liquid by a block of polymethylmethacrylate (PMMA) plastic (SOS $\left.c_{\mathrm{P}}, \mathrm{Al} Z_{\mathrm{P}}, \mathrm{AC} \alpha_{\mathrm{P}}(f)\right)$ and using the same transducers as in the flowmeter in Fig. $1 \mathrm{a}$ ). The motivation behind using the plastic is that no liquid must be handled and that the testing is performed under more reliable and reproducible conditions. a)
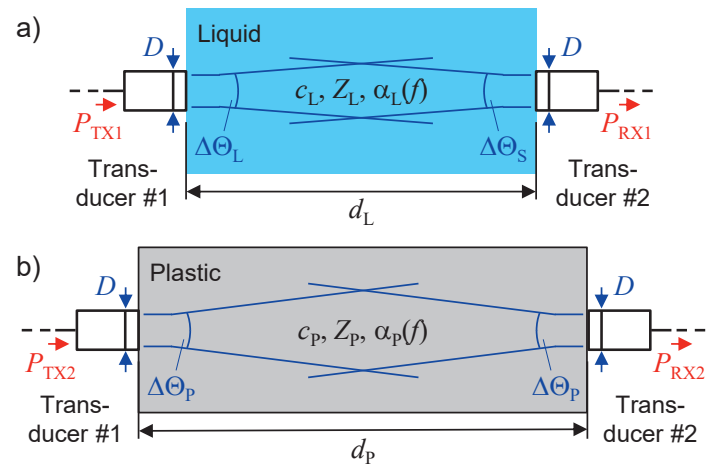

Fig. 1. Ultrasound transmission paths: a) Flowmeter (liquid), b) hardware simulator (plastic block).

In the following, the two setups in Fig. 1 are compared to each other by theoretically analyzing the characteristics of both different ultrasound transmission paths. Finally, the proposed concept has been experimentally evaluated and according measurement results are presented.

\section{Ultrasound Transmission Path}

For the same absolute TOF, the distance $d_{\mathrm{P}}$ between the transducers in the plastic must be chose as follows (distance $d_{\mathrm{L}}$ in the liquid):

$d_{\mathrm{P}}=d_{\mathrm{L}} \cdot c_{\mathrm{P}} / c_{\mathrm{L}}$

Using the same transducers (aperture diameter $D$, frequency $f$ ) in both setups, the angular beam widths $\Delta \theta_{\mathrm{L}}$ and $\Delta \theta_{\mathrm{P}}$, respectively, are different:

$\Delta \theta \sim \frac{c}{D \cdot f}, \quad \Delta \theta_{\mathrm{P}}=\Delta \theta_{\mathrm{L}} \cdot \frac{c_{\mathrm{P}}}{c_{\mathrm{L}}}$

Accordingly, the transmission attenuation $a_{\mathrm{US}}$ also depends on the SOS $c$ (transmit and receive 
powers $P_{\mathrm{TX}}$ and $P_{\mathrm{RX}}$; aperture and electrical efficiencies $\eta_{\mathrm{ap}}$ and $\eta_{\mathrm{el}}$, respectively; $\left.\mathrm{AC} \alpha(f)\right)$ [1]:

$a_{\mathrm{US}}=\frac{P_{\mathrm{TX}}}{P_{\mathrm{RX}}}=\frac{16 \cdot c^{2} \cdot d^{2}}{\pi^{2} \cdot D^{4} \cdot f^{2} \cdot \eta_{\mathrm{ap}}^{2} \cdot \eta_{\mathrm{el}}} \cdot \mathrm{e}^{2 \cdot \alpha(f) \cdot d}$

The acoustic coupling between the transducers and the propagation medium depends on the matching between the Als of both. Likewise, the decay of the transducer 'ringing', i.e. multiple reflections inside the transducer, over TOF depends on the matching. With the hardware simulator, the ringing is expected to decay faster compared to the flowmeter, because of the better matching of the plastic.

\section{Measurement Results}

The two different setups have been tested using water $\left(d_{\mathrm{L}}=55 \mathrm{~mm}, c_{\mathrm{L}}=1480 \mathrm{~m} / \mathrm{s}, Z_{\mathrm{L}}=1.48 \mathrm{MRayl}\right)$ and PMMA plastic $\left(d_{\mathrm{P}}=100 \mathrm{~mm}, c_{\mathrm{P}} \approx 2700 \mathrm{~m} / \mathrm{s}\right.$, $Z_{\mathrm{P}} \approx 3.2 \mathrm{MRayl}$ ). The same transducers $\left(f_{0}=2 \mathrm{MHz}\right.$ center frequency, $D=10 \mathrm{~mm}$ aperture diameter) have been used in both cases. Transmission measurements have been performed with a calibrated vector network analyzer (model Bode 100; OMICRON electronics $\mathrm{GmbH}$, Klaus, Austria). Time domain transmission signals $s_{21}(t)$ have been calculated from the measured frequency domain transmittances $S_{21}(f)$ by means of the inverse Fourier transform [2]. In Fig. 2, the measured transmission signals are shown:

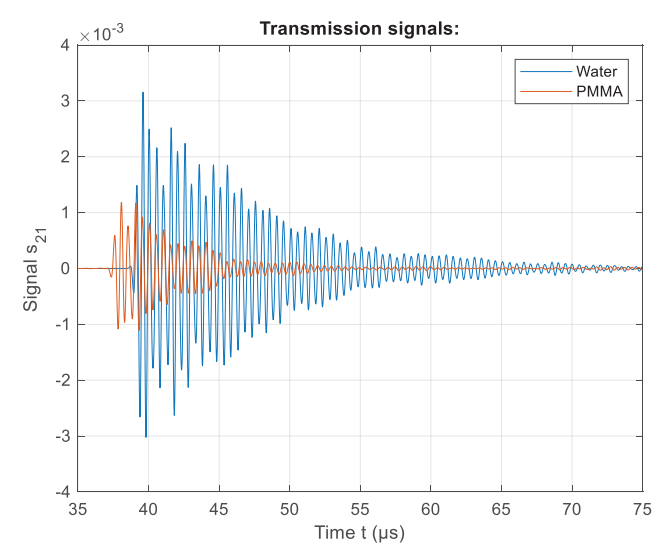

Fig. 2. Measured transmission signals: Water (blue), PMMA block (red).

As can be seen, almost the same TOFs are given, as the distance $d_{\mathrm{L}}$ has been chosen according to (1). Both signals have a very similar shape, except of the different amplitudes and decays over TOF. With the different SOSs, the transmission attenuation of the hardware simulator is expected to be $5.22 \mathrm{~dB}$ larger compared to with the water path, see (3). The envelopes of the measured transmission signals over TOF in Fig. 3 show an amplitude difference of $8.4 \mathrm{~dB}$. This difference is because the AC of the PMMA is significantly larger compared to that of the water, and so the transmission attenuation in (3).

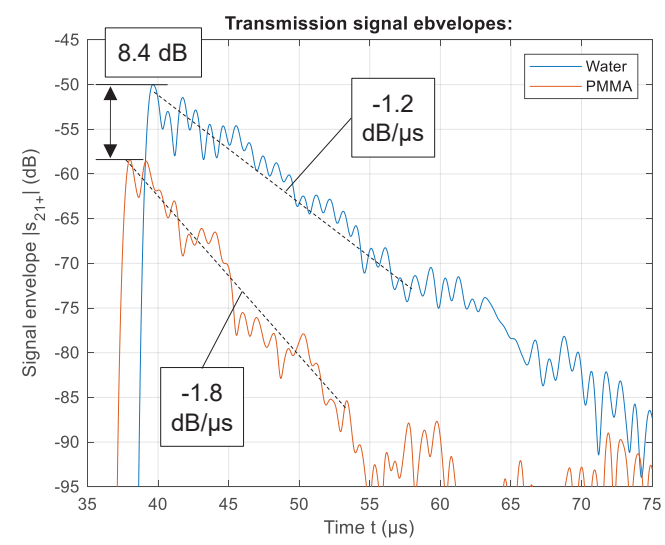

Fig. 3. Envelopes of measured transmission signals: Water (blue), PMMA block (red).

The transmission signal from the water path decays with about $1.2 \mathrm{~dB} / \mu$ s over TOF. With the PMMA, a faster decay of about $1.8 \mathrm{~dB} / \mu$ s is given. This difference is found, because the decay of the transducer ringing is the faster the better the acoustic matching between the sound propagation medium and the transducer is. The $\mathrm{Al}$ of the PMMA is more than twice large as the Al of water. Accordingly, the hardware simulator is much better matched to the high Al of the transducer.

\section{Summary and Conclusions}

In this contribution, a hardware simulator with PMMA as sound propagation medium for testing the sensor electronics of ultrasonic flowmeters has been presented and discussed. Measurements with a vector network analyzer have been performed to compare this setup with the flowmeter containing water. Transmission signals in both cases show a similar shape but different amplitudes. The latter is because of the different transmission attenuation linked to the different SOSs and the different attenuation coefficients. Furthermore, both transmission signals show different decays over TOF, what is caused by the different matching between the sound propagation medium and the transducers in the two cases. The findings from the experimental evaluation of the concept are in good agreement with expectations from the theoretical modeling. The realized hardware simulator allows for reliable transmission signal measurements with featuring well-defined conditions.

\section{References}

[1] B.R. Tittmann, Ultrasonic Sensor Viscosity of Mineral Oil for Built-in Pipe Applications, Sensor+Test Conf., 171-176 (2011); doi: 10.5162/sensor11/a7.3

[2] M. Vogt, H. Ermert, Quantitative Analysis of Liquids and Emulsions by Means of High-Frequency Ultrasound (15-35 MHz), IEEE Ultrason. Symp., 68-71 (2011); doi: 10.1109/ULTSYM.2011.0018 